\title{
Impact of Neuroradiology-Based Peer Review on Head and Neck Radiotherapy Target Delineation
}

\author{
(D) S. Braunstein, (D) C.M. Glastonbury, (D). Chen, (D).M. Quivey, and (D)S.S. Yom
}

\begin{abstract}
BACKGROUND AND PURPOSE: While standard guidelines assist in target delineation for head and neck radiation therapy planning, the complex anatomy, varying patterns of spread, unusual or advanced presentations, and high risk of treatment-related toxicities produce continuous interpretive challenges. In 2007, we instituted weekly treatment planning quality assurance rounds as a joint enterprise of head and neck radiation oncology and neuroradiology. Here we describe its impact on head and neck radiation therapy target delineation.
\end{abstract}

MATERIALS AND METHODS: For 7 months, treatment planning quality assurance included 80 cases of definitive (48\%) or postoperative (52\%) head and neck radiation therapy. The planning CT and associated target volumes were reviewed in comparison with diagnostic imaging studies. Alterations were catalogued.

RESULTS: Of the 80 cases, 44 (55\%) were altered, and of these, $61 \%$ had clinically significant changes resulting in exclusion or inclusion of a distinct area or structure. Reasons for alteration included the following: gross or extant tumor, 26/44 (59\%); elective or postoperative coverage, 25/44 (57\%); lymph nodes, 13/44 (30\%); bone, 7/44 (16\%); skull base, 7/44 (16\%); normal organs, 5/44 (11\%); perineural, 3/44 (7\%); distant metastasis, $2 / 44$ (5\%); and eye, $1 / 44(2 \%)$. Gross tumor changes ranged from $0.5 \%$ to $133.64 \%$, with a median change in volume of 5.95 $\mathrm{mm}^{3}(7.86 \%)$. Volumes were more likely to be increased $(73 \%)$ than decreased $(27 \%)$.

CONCLUSIONS: A collaborative approach to head and neck treatment planning quality assurance has an impact. Cases likely to have challenging patterns of infiltrative, intracranial, nodal, orbital, or perineural spread warrant intensive imaging-based review in collaboration with a diagnostic neuroradiologist.

ABBREVIATIONS: $\mathrm{CTV}=$ clinical tumor volume; $\mathrm{GTV}=$ gross tumor volume; $\mathrm{HN}=$ head and neck; $\mathrm{IMRT}=$ intensity-modulated radiation therapy; $\mathrm{PTV}=$ planning target volume; TPQA = treatment planning quality assurance

$\mathbf{R}^{\mathrm{ctang}}$ etrospective and prospective studies demonstrate increased efficacy from multidisciplinary physician interaction, ${ }^{1,2}$ and team-based approaches to patient care are routine within radiation oncology. However, the process of radiation therapy target delineation remains an essentially solitary activity, and the impact of collaborative peer review is a contested issue. One survey suggested that major alterations from this type of process were rare, occurring in $<6 \%$ of head and neck (HN) plans, though the ex-

Received November 17, 2014; accepted after revision August 17, 2016.

From the Departments of Radiation Oncology (S.B., C.M.G., J.C., J.M.Q., S.S.Y.) and Radiology (C.M.G.), University of California, San Francisco, San Francisco, California.

Paper previously presented in part at: Annual Meeting of the American Society of Radiation Oncology, October 2-6, 2011; Miami, Florida.

Please address correspondence to Sue S. Yom, MD, PhD, UCSF Radiation Oncology, 1600 Divisadero St, Suite H-1031, San Francisco, CA 94143-1708; e-mail: yoms@radonc.ucsf.edu

http://dx.doi.org/10.3174/ajnr.A4963 tent of alterations was noted to be dependent on the reviewing peer's subsite experience level. ${ }^{3}$

The weakness of these studies as applied to HN cancer stems from a tendency to underestimate the specialized nature of anatomically defined $\mathrm{HN}$ radiation therapy and its unique interdependence with neuroradiology. Head and neck malignancies comprise a heterogeneous group of neoplasms characterized by complex local and regional anatomy, varying patterns of spread, and frequent occurrence of unusual and/or advanced presentations. Acquiring proficiency in the interpretation of $\mathrm{HN}$ imaging is difficult due to the subtlety of the characteristics that may suggest benign or malignant disease and distinguishing them from normal or inflamed tissue. Because management frequently consists of staged, multimodal combinations of surgery, systemic therapy, and/or radiation therapy, the interpretation of sequential image sets is exceptionally challenging, particularly the discrimination of posttreatment changes from residual disease. ${ }^{4}$ Previous studies have found that after re-interpretation by a specialist head 


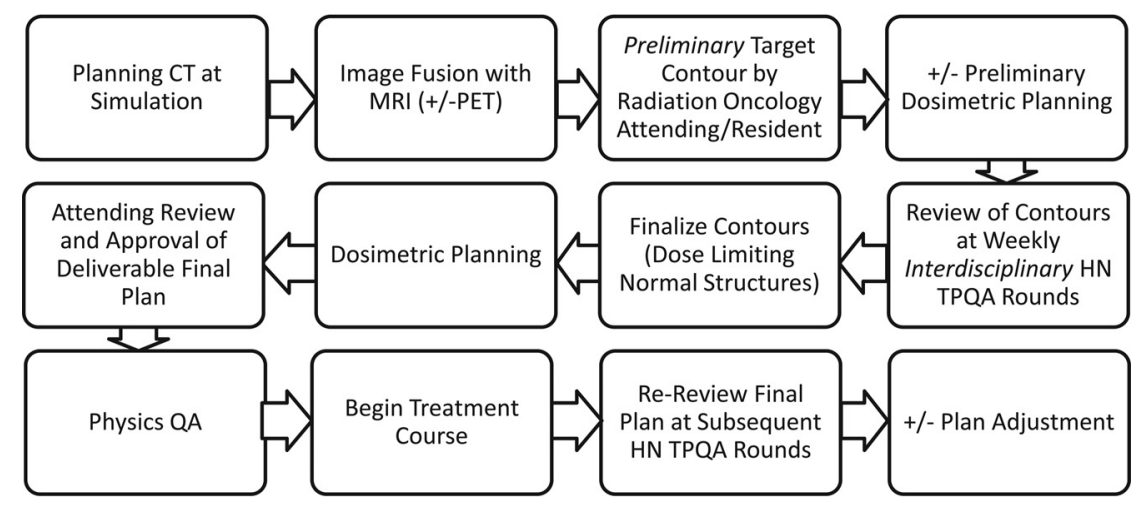

FIG 1. Treatment planning workflow for head and neck radiation oncology.

and neck neuroradiologist, changes in staging or management occur in $38 \%-56 \%$ of cases. ${ }^{5,6}$

Beginning in 2007, diagnostic neuroradiology participation was included as part of weekly $\mathrm{HN}$ treatment planning quality assurance (TPQA) rounds at our institution. The format includes diagnostic imaging review for new and follow-up patients, as well as the highly prioritized review of proposed radiation therapy target volumes and normal organ delineations, which are peerreviewed by $\mathrm{HN}$ radiation oncology and neuroradiology physicians. As of June 2010, electronic documentation was prepared pre- and post-TPQA. This study characterizes the impact of diagnostic neuroradiology involvement on the radiation therapy planning process.

\section{MATERIALS AND METHODS HN TPQA Workflow}

The HN TPQA team consists of radiation oncologists (including S.S.Y., J.M.Q.); diagnostic neuroradiologists (including C.M.G.); neuroradiology and radiation oncology students, residents, and fellows; and members of the dosimetry and physics teams (including J.C.). Review includes plans intended for treatment of the upper aerodigestive tract, and thyroid and $\mathrm{HN}$ soft-tissue and lymphatic and cutaneous regions. Pediatrics, spine, mediastinal, and lung cancer cases are occasionally reviewed, but these were excluded from this report. Figure 1 describes the treatment planning workflow.

Before TPQA, radiation planning CT scans are acquired with 3-mm section resolution; 1.5-mm section thickness is acquired for stereotactic cases. The planning CT is fused in multiple orientations with all available imaging studies, including MR imaging, diagnostic CT, and PET/CT. Target volumes and pertinent normal organ structures are delineated in advance by the radiation oncology team, reviewed and approved by the attending radiation oncologist who is designated by identifying first and last initials, and saved as a "Pre-TPQA" file.

Per the International Commission on Radiation Units and Measurements report $50,{ }^{7}$ targets are designated as gross tumor volume (GTV), which includes all gross tumor appreciated on clinical examinations or visualized on imaging; clinical tumor volume (CTV), denoting regions of clinical risk outside the GTV, which include both areas considered at highest risk for involvement (CTV1) and those considered at lesser risk such as prophylactically included elective nodal basins (CTV2); and planning target volume (PTV), which adds a small margin to each CTV to account for uncertainties of daily patient and machine setup. A dose range of 6000-7400 cGy is designated for the highest dose target and/or involved or high-risk nodal volumes (GTV, CTV1); and typically 4500-6000 cGy is designated for CTV2 (elective CTV). In the practice of our institution, CTV1 and CTV2 volumes are frequently subdivided (eg, CTV1 could be subdivided into CTV_6996 and CTV_6600, to clarify relative levels of highest risk by using the radiation dose as the suffix [given in units of centigray] and to enable "dose painting" when creating the radiation therapy plan). PTV volumes are created as a 2- to 3-mm expansion from the CTV; PTVs are prescribed at the dose of their corresponding CTVs and are used in the final development, optimization, and evaluation of the computerized radiation plan. Image fusion and delineations are usually performed on MIM (MIM Software, Cleveland, Ohio), though stereotactic cases are reviewed on a Multiplan (Accuray, Sunnyvale, California) station. Dosimetry is performed on Pinnacle (Phillips Healthcare, Best, the Netherlands), TomoTherapy (Accuray), or Multiplan systems. An example of image fusion is shown in Fig 2.

TPQA occurs at a workstation complex that includes juxtaposed Pinnacle, TomoTherapy, MIM, and PACS terminals, enabling a parallel display of all imaging and target delineation data. Following review of the clinical, surgical, and pathologic details of the case, the neuroradiologists review all available diagnostic imaging. Preliminary target volumes are then reviewed on the MIM, Pinnacle, or TomoTherapy station, superimposed on the radiation planning CT and all fused diagnostic imaging studies. This process is characterized by interactive discussion and repeat viewing of the diagnostic imaging. The primary focus is on delineation of gross disease and areas of high clinical risk but also includes consideration of sparing adjacent uninvolved and/or critical structures. Any proposed changes are carried out in consultation with the neuroradiologist. After collaborative target volumes are created, a postreview structure set is saved as "Post-TPQA." A description of alterations is catalogued in the electronic medical record (MOSAIQ; Elekta, Stockholm, Sweden).

\section{Sample Population and Statistical Analysis}

Approval was granted for review of demographic and radiographic data by the Committee on Human Research. A primary set of $80 \mathrm{HN}$ consecutive treatment plans was reviewed at TPQA from August 2010 to February 2011; this era was selected for study as quality assurance documentation was consistent by this time point and it was approximately at the midpoint of our quality assurance experience. Descriptive information regarding the type of change by anatomic compartment was documented at the time of review. Volumetric information was later quantitatively compared between the Pre-TPQA and Post-TPQA files. Alterations in major disease-related targets (GTVs, CTVs) and anatomic compartments (soft tissue, lymph nodes, perineural invasion, bone invasion) were recorded. The significance of the frequency and 



FIG 2. Example of image fusion performed at TPQA. Green crosshairs intersect over the laryngeal tumor. Rows represent the following: radiation oncology treatment planning CT scan, diagnostic PET, diagnostic MR imaging, fusion of treatment planning CT with PET, and fusion of treatment planning CT with MR imaging.

volume of change among HN subsites was analyzed by $\chi^{2}$ contingency analysis and 1-tailed analysis of variance, respectively.

To rule out the presence of a "learning curve," we similarly reviewed a second set of $40 \mathrm{HN}$ consecutive treatment plans (August 2011 to November 2011). A 2-tailed Fisher exact test was used to compare the extent of target changes between the 2 sets.

\section{RESULTS}

\section{Case Characteristics}

Demographic and histopathologic information for the 80 consecutive cases is presented in Table 1. Patient median age was 49 years (range, 29-89 years). Predominant subsites included the oral cavity (24\%), oropharynx (18\%), sinonasal region (11\%), and nasopharynx (10\%). Additional subsites were skin (9\%), salivary gland (9\%), hypopharynx (6\%), and larynx (5\%). "Other" (9\%) subsites included the orbit, lacrimal gland, maxilla, and neck (Table 1). Squamous cell carcinoma was the dominant histology (76\%), followed by adenocarcinoma (5\%) and lymphoma (4\%). Other (15\%) histologies included sarcoma, esthesioneuroblastoma, neuroendocrine and mucoepidermoid carci- noma, and ameloblastoma (Table 1). Most cases were advanced-stage (60\% stage IV, 19\% stage III). Forty-eight percent of patients had definitive radiation-based treatment, and $52 \%$ had adjuvant radiation therapy following surgery.

\section{Types of Change during TPQA}

Overall, 55\% (44/80) of Pre-TPQA structure sets were changed. The frequency of changes across subsites is shown in Fig $3 A$, from the hypopharynx altered in $80 \%$ (4/5) of cases to "other" with 29\% (2/7) altered. Alterations were considered "clinically significant" if they resulted in exclusion or inclusion of a distinct area or structure and would change the radiation therapy plan with potential impact on disease control or toxicity. Sixty-one percent of altered plans (34\% of all cases) had clinically significant changes, as shown in Fig $3 B$. Thirty-two percent $(26 / 80)$ of cases had changes in the GTV, and 31\% (25/80) had changes in the elective CTV (Fig $3 B)$. Only 10 of 44 altered cases (23\%) had changes in both the GTV and elective CTV. Other changes included addition or subtraction of lymph nodes $(16 \%, 13 / 80)$, delineation of perineural pathways of spread $(4 \%, 3 / 80)$, or reassessment of cancerous bone invasion $(9 \%, 7 / 80)$. Specific forms of perineural and bony/cartilaginous target volume alteration most frequently involved the inclusion or exclusion of branches of the trigeminal and facial cranial nerves and skull base perineural invasion, such as minor branches around the pterygopalatine fossa and cavernous sinus or Meckel cave, and fine editing of structures such as the clivus, mandible, sinuses, hyoid, laryngeal cartilages, and trachea. For postoperative cases, detailed editing was frequently performed around the areas of reconstruction and flap placement. Frequency and general categorization of types of changes were similar among definitive and postoperative patients.

\section{Volumetric Assessment of Alterations during TPQA}

GTV and elective CTV changes are presented in Table 2. For altered plans, the mean absolute volume change for GTV was 14.88 $\mathrm{mm}^{3}$ (19.75\%), and for CTV, it was $14.63 \mathrm{~mm}^{3}$ (21.83\%). As shown in Fig $4 A$, most changes were increases in GTV and elective CTV (approximately two-thirds of changed plans). As shown in Fig $4 B$, both GTV and CTV changes were heterogeneous, ranging from $<1 \%$ to $>100 \%$ (up to $275 \%$ for elective CTV changes). Furthermore, there was no difference by subsite in the frequency of overall $(n=80)$ volumetric changes in GTV or CTV (1-tailed 
Table 1: Patient demographic and histopathologic information

\begin{tabular}{lc}
\hline & No. (\%) \\
\hline Total No. of patients & $80(100)$ \\
Sex & \\
M & $59(74)$ \\
F & $21(26)$ \\
Mean/median age (range) (yr) & $51 / 49(29-89)$ \\
Anatomic subsite & \\
Oral cavity & $19(24)$ \\
Oropharynx & $14(18)$ \\
Sinonasal & $9(11)$ \\
Nasopharynx & $8(10)$ \\
Skin & $7(9)$ \\
Salivary gland & $7(9)$ \\
Other & $7(9)$ \\
Hypopharynx & $5(6)$ \\
Larynx & $4(5)$ \\
Histology & \\
Squamous cell carcinoma & $61(76)$ \\
Adenocarcinoma & $4(5)$ \\
Lymphoma & $3(4)$ \\
Other & $12(15)$ \\
Stage & \\
I & $4(5)$ \\
II & $13(16)$ \\
III & $15(19)$ \\
IV & $48(60)$ \\
Radiotherapy intent & $38(48)$ \\
Definitive & $42(52)$ \\
Postoperative &
\end{tabular}

ANOVA, $P=.64$ and $P=.74$, respectively). Examples of alterations are shown in Fig 5.

In the 40-patient follow-up set, the frequency of change remained stable at $45 \%(18 / 40)$ compared with $55 \%$ in the initial cohort (Fisher exact test, $P=.34$ ). As shown in Fig 6, the categorization of changes was extremely similar to that of the earlier cohort.

\section{DISCUSSION}

In this report, approximately half of all radiation therapy plans were edited during neuroradiology-based peer review, and clinically significant changes were seen across all anatomic subsites and in both definitive and postoperative patients. The level of change averaged approximately $25 \%$ in volume but varied widely by patient, with reduction of target volumes (and presumably toxicity) in one-third of patients. Small GTV changes were sometimes highly clinically significant, especially for cases of lymph node involvement, osseous infiltration, or perineural extension. In postoperative patients, neuroradiologist input was valuable in identifying areas of close margin or routes of microscopic disease potentially underappreciated at the time of surgery. Because volumes at risk for microscopic disease were reviewed for all definitive and postoperative cases, the CTVs had a high frequency of change.

These findings confirm the impact that neuroradiology-based peer review has on the delineation of $\mathrm{HN}$ radiation therapy target volumes. Previous studies of collaboration between subsitespecific radiation oncologists and radiologists have suggested similar findings. In 1 study, a panel reviewed tumor delineations of 10 patients with non-small cell lung cancer. ${ }^{8}$ The radiation
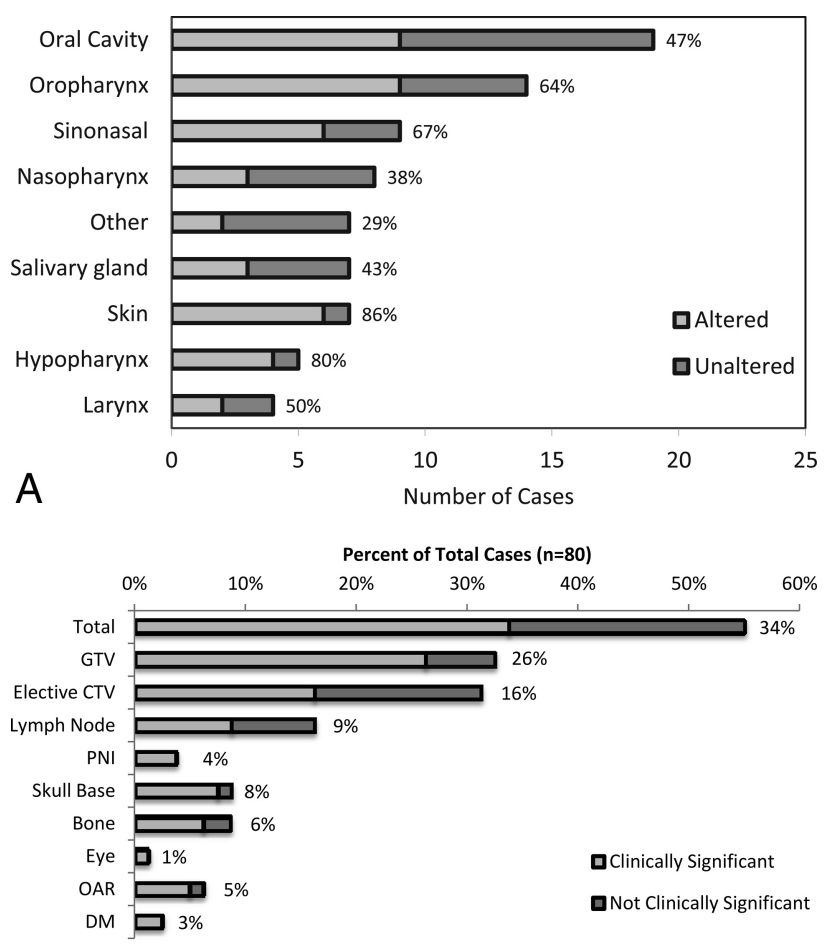

B

FIG 3. A, Alterations by subsite following HN TPQA. Light gray bars represent cases that were altered after review. Dark gray bars represent cases that were reviewed without subsequent alterations. Percentages to the right of the bars represent the fraction of cases with alterations. B, Types of treatment plan alterations. Light gray bars and data labels represent the percentage of total plans $(n=80)$ found to have changes by listed category. Dark gray bars represent the fraction of total plans with nonsignificant changes. PNI indicates perineural invasion; OAR, organs at risk; and DM, distant metastases. Note that $x$-axes were broken to reduce the width of the figure while maintaining a display of low and high values.

Table 2: Absolute volumetric changes in GTV and CTV from preTPQA to post-TPQA in altered plans

\begin{tabular}{lcc}
\hline & $\begin{array}{c}\text { Volume } \\
\left(\mathbf{m m}^{3}\right)\end{array}$ & $\begin{array}{c}\text { Percentage } \\
\text { Change }\end{array}$ \\
\hline GTV $(n=26$ cases $)$ & 14.88 & +19.75 \\
Mean & 5.95 & +7.86 \\
Median & $1.95-130.59$ & $0.50-133.64$ \\
Abs. range & & \\
CTV $(n=25$ cases $)$ & 14.63 & +21.83 \\
Mean & 10.34 & +2.50 \\
Median & $1.84-112.70$ & $0.5-275.68$ \\
Abs. range & &
\end{tabular}

Note:-Abs. indicates absolute.

oncologists' average GTVs were $>33 \%$ larger and more heterogeneous, outcomes resulting from a lower level of proficiency in applying window settings, discriminating tumor from consolidation, identifying involved lymph nodes, recognizing partial volume effects, and identifying pleural and chest wall involvement. ${ }^{8,9}$ Similarly, Horan et $\mathrm{al}^{10}$ reported GTV delineation for 10 patients with cancer by a radiologist and 2 radiation oncologists. Two of 5 cases of HN cancer showed major discordance. Discrepancy was attributed to disparate access to clinical information and diagnostic imaging expertise. A follow-up prospective study of non-small cell lung cancer radiation therapy plans included a formal collaborative session to finalize target volumes. ${ }^{11}$ Changes occurred in 


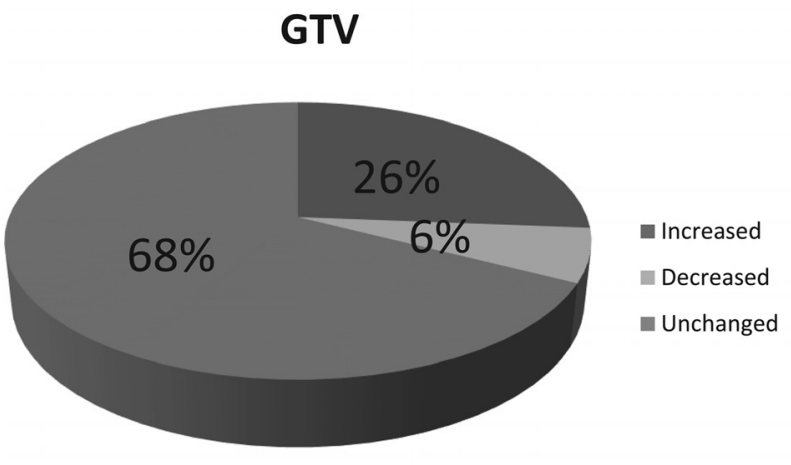

CTV

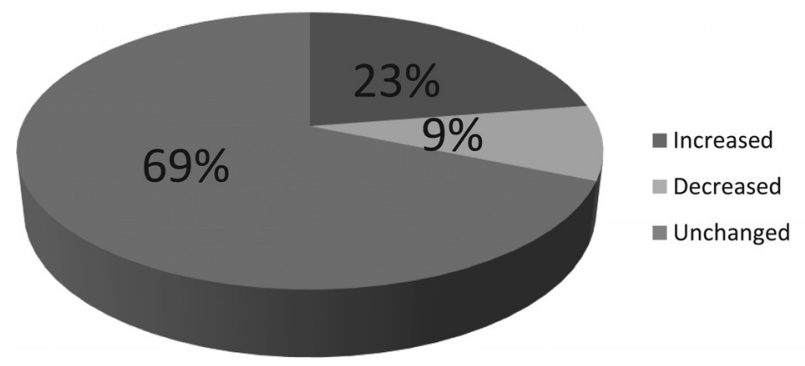

A

B

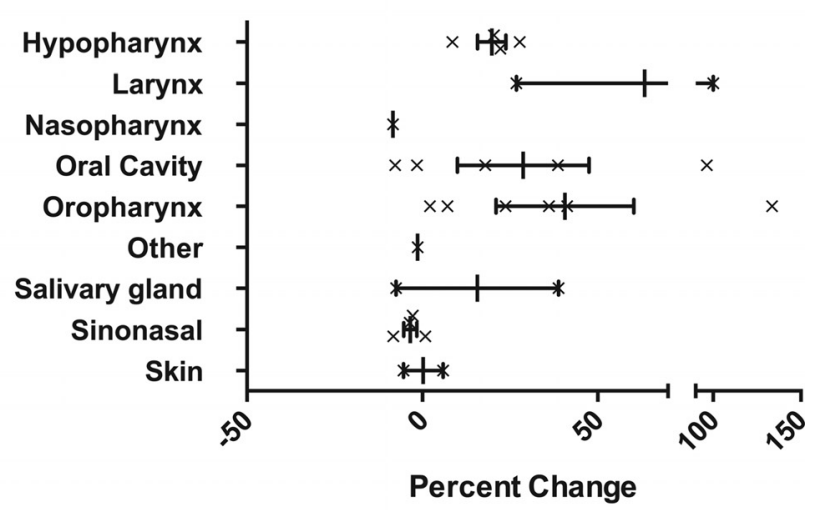

CTV Changes $(n=25)$

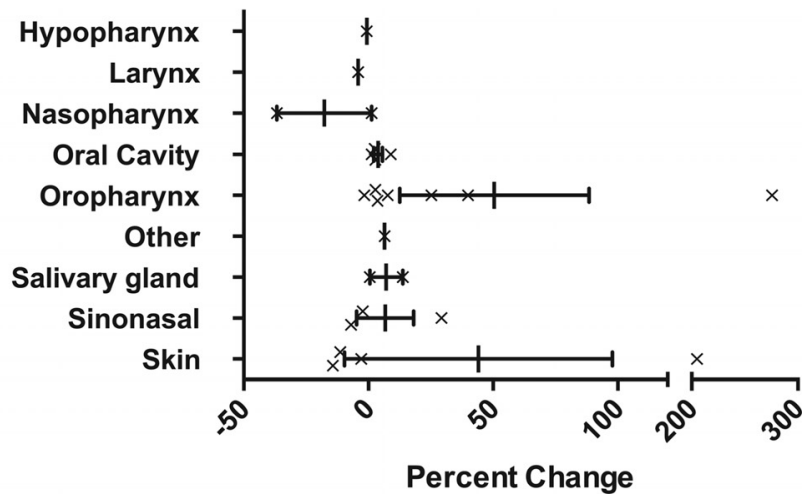

FIG 4. A, Volumetric changes in Pre-TPQA plans compared with Post-TPQA. Overall direction of GTV and CTV change. $B$, Volumetric changes in Pre-TPQA plans compared with Post-TPQA. Percentage of volume changes by subsite.

19 of 20 cases, with radiation oncologists reporting greater confidence in the resultant GTVs.

The introduction of highly conformal treatments such as intensity-modulated radiation therapy (IMRT) has increased the anatomic specificity of $\mathrm{HN}$ radiation therapy targeting, with the potential to decrease toxicities and adverse events. ${ }^{12,13}$ However, because of this increased precision, small errors in treatment design and delivery can affect clinical outcomes. ${ }^{14}$ Thus, major societies have issued guidelines enumerating aspects of the quality assurance process for IMRT, ${ }^{15}$ and most academic radiation oncology institutions use peer review to improve planning consistency. ${ }^{16}$ While textbooks, anatomic atlases, ${ }^{17,18}$ and evolving auto-segmentation tools ${ }^{19}$ are available to assist the individual practitioner, process studies have demonstrated a continuing need for multidisciplinary integration to assure effective radiation therapy planning. ${ }^{20}$

Even among diagnostic neuroradiologists, interpretation of $\mathrm{HN}$ imaging is recognized as a challenging area that requires special effort in education and secondary consultation. ${ }^{21}$ Regarding radiation oncology, while it developed as a subspecialty of radiology in the first half of the 20th century, ${ }^{22}$ the residency now includes no formal diagnostic radiologic education. Meanwhile, developments in imaging acquisition and manipulation have led to increasing complexity and specificity of $\mathrm{HN}$ target delineation. Notably, the development of novel MR imaging and PET se- quences has produced increasingly sophisticated imaging data for review. ${ }^{23,24}$ Integrating these sequences requires fusion to the radiation-planning CT for maximal utility, and thus many radiation oncologists now oversee complex workflows involving multimodality imaging fusion. These processes may require oversight and adjustment, but quality assurance of these procedures is not routine. $^{25,26}$

Radiation oncologists collaborate with urologists in prostate brachytherapy delivery ${ }^{27}$ and with neurosurgeons in designing stereotactic radiosurgery for the brain. ${ }^{28}$ However, while the benefits of collaboration with diagnostic radiology have been promoted for both external beam ${ }^{8}$ and brachytherapy ${ }^{29}$ treatment planning, there is little evidence of formal inclusion in these spheres. ${ }^{9}$ Cited barriers include distinct workflows, separate locations, independent computer systems, and lack of defined billing mechanisms. ${ }^{30}$ Nonetheless, rigorously reviewed treatment planning is an essential component of care for patients with $\mathrm{HN}$ cancer because salvage options after inadequate radiation therapy are limited. Our experience documents the impact of collaboration across these formidable logistical barriers.

There are limitations to this study. Because of uncertainty independent of the target delineation process (patient setup, machine-based physical uncertainties, multidirectional misalignments), CTVs are further expanded during radiation therapy planning to create planning treatment volumes (PTVs), which are 



FIG 5. Treatment planning examples of TPQA changes. Red represents the pre-TPQA GTV or CTV1, and orange represents the altered post-TPQA GTV or CTV1. Yellow represents the preTPQA or CTV2, and blue represents the altered post-TPQA or CTV2. Changes include the following: increase in GTV for suspicion of gross disease involving the hyoid bone $(A)$, increased GTV and CTV for pre-mastoid disease (B), expansion of CTV to include suspected PNI within the pterygopalatine fossa $(C)$, and additional nodal GTV but decreased primary tumor GTV to spare additional laryngeal toxicity $(D)$. PNI indicates perineural invasion.

\section{Changes by Type}

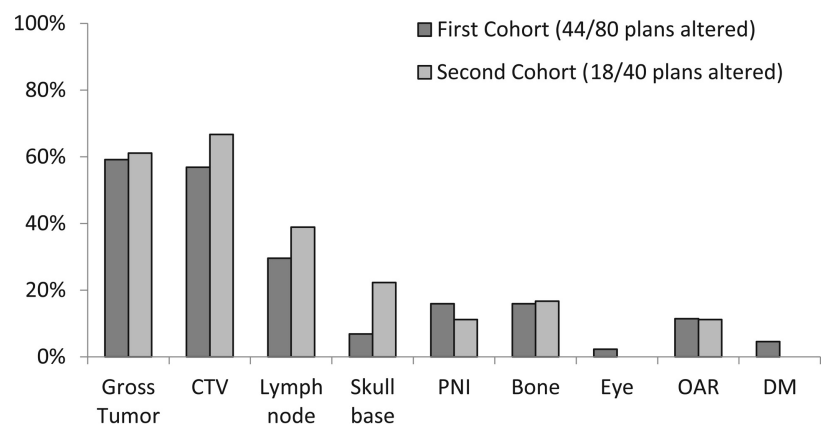

FIG 6. Comparison of TPQA results from 2 different time points. There is an extremely similar frequency of types of changes in the initial cohort $(n=80)$ and the follow-up cohort a year later $(n=40)$. PNI indicates perineural invasion; OAR, organs at risk; and DM, distant metastases.

used to design the final delivered plan and may suppress the effect of small changes in GTVs and CTVs. ${ }^{31,32}$ However, increasingly advanced radiation therapy delivery that decreases these uncertainties has led many practitioners to reduce PTV margins, amplifying the impact of small changes in tumor delineation. ${ }^{10}$ Furthermore, we designated clinically significant changes as those that would have resulted in omission or inclusion of an anatomically distinct area; this level of difference would not necessarily be remedied by PTV expansions.

Another limitation may relate to the expectation of scheduled review, with the possibility that the radiation oncologists postponed decisions on difficult questions until TPQA, resulting in many changes. This phenomenon probably did occur to some extent, but we chose to incorporate these tendencies. Changes reflecting questioning or uncertainty are as important in their need for review as areas of unrecognized error. We believe these "gray areas" are better included than excluded from TPQA.

Third, because of the fluid nature of TPQA, we could not isolate changes made at the discretion of the neuroradiologists versus those suggested by other participants. In fact, the presence of trainees often led to explanations of clinical insights with ramifications for target delineation. A less easily quantified value of TPQA is the educational and team-building function, which increases the capacities of the group as a whole across time. This is a by-product uniquely stemming from the involvement of neuroradiology in the target delineation process.

Finally, it is possible that TPQA was evolutionary and the frequency of alterations changed with time. However, a review of cases from a later period yielded a similar frequency of changes, suggesting that the impact of the review did not diminish. Informally, we note that approximately half of our cases continue to be altered in some manner at TPQA rounds to this day.

The intensity with which our process is conducted, in a concentrated, uninterrupted period of dedicated time each week, differentiates TPQA from informal arrangements and enabled a concrete documentation of the benefits of collaboration. At many high-volume HN programs, radiation oncologists may query a neuroradiologist about a specific aspect causing concern or confusion. In our TPQA process, neuroradiology is intricately involved in the inspection of targets through their superior-toinferior extent by using comprehensive pre-prepared image fusion sets with targets overlaid on them. Inevitably, novel questions are raised by this convergence of information. Due to this sort of repeat exposure, our neuroradiology team is now experienced in the challenges of the radiation oncology decision-making process (because describing a tumor is not at all the same as drawing it), and they can understand and discuss the clinical trade-offs that are incurred related to specific targets of high- and low-dose prescription. The repeat synergy of experts at TPQA creates a network of knowledge that incorporates not only purely radiologic viewpoints but others that uniquely arise from the convergence of radiation therapy and radiology. While aspects of this level of teamwork may be replicated in ad hoc arrangements, we believe that structured interactions enabled this synergy at the highest level. 
As a by-product of this process, there are some additional clinical benefits of interdisciplinary case review. Occasionally, further evolution or early recurrence of disease was identified on planning CT scans, leading to changes in management. ${ }^{10}$ Diagnostic MR images and PET/CT scans were sometimes obtained elsewhere, and TPQA helped overcome the limitations of suboptimal imaging and provided education for participants about appropriate imaging protocols. Last, within the TPQA framework, selected patients' imaging changes could be reviewed during a radiation therapy course which lasts several weeks, with the opportunity to re-plan the radiation treatment due to changes in anatomy or setup. ${ }^{33}$ For the neuroradiologists, TPQA provided a focused exposure to imaging correlates of radiation therapy treatment response and sequelae $\mathrm{e}^{34}$ and a repertoire of pertinent information to include in reports to assist with radiation therapy target delineation. ${ }^{9,35,36}$

\section{CONCLUSIONS}

Structured collaborative review of radiation therapy target delineation promotes the most effective use of diagnostic imaging in head and neck radiation oncology treatment planning. Interactions with diagnostic neuroradiology should be maximized, to promote a high level of treatment quality in the face of a proliferating array of complex imaging tools.

Disclosures: Sue S. Yom-UNRELATED: Grants/Grants Pending: Genentech, National Comprehensive Cancer Network Foundation, Comments: funding for clinical trials*; Other: American Society for Radiation Oncology, Comments: honoraria for journal editorship. *Money paid to the institution.

\section{REFERENCES}

1. Wheless SA, McKinney KA, Zanation AM. A prospective study of the clinical impact of a multidisciplinary head and neck tumor board. Otolaryngol Head Neck Surg 2010;143:650-54 CrossRef Medline

2. Nguyen NP, Vos P, Lee H, et al. Impact of tumor board recommendations on treatment outcome for locally advanced head and neck cancer. Oncology 2008;75:186-91 CrossRef Medline

3. Lefresne S, Olivotto IA, Joe $\mathrm{H}$, et al. Impact of quality assurance rounds in a Canadian radiation therapy department. Int J Radiat Oncol Biol Phys 2013;85:e117-21 CrossRef Medline

4. Saito N, Nadgir RN, Nakahira M, et al. Posttreatment CT and MR imaging in head and neck cancer: what the radiologist needs to know. Radiographics 2012;32:1261-82; discussion 1282-84 CrossRef Medline

5. Loevner LA, Sonners AI, Schulman BJ, et al. Reinterpretation of cross-sectional images in patients with head and neck cancer in the setting of a multidisciplinary cancer center. AJNR Am J Neuroradiol 2002;23:1622-26 Medline

6. Lysack JT, Hoy M, Hudon ME, et al. Impact of neuroradiologist second opinion on staging and management of head and neck cancer. J Otolaryngol Head Neck Surg 2013;42:39 CrossRef Medline

7. Jones D. ICRU Report 50-Prescribing, Recording and Reporting Photon Beam Therapy. Med Phys 1994;21:833 CrossRef

8. Giraud P, Elles S, Helfre S, et al. Conformal radiotherapy for lung cancer: different delineation of the gross tumor volume (GTV) by radiologists and radiation oncologists. Radiother Oncol 2002;62: 27-36 CrossRef Medline

9. Roy AE, Wells $\mathrm{P}$. Volume definition in radiotherapy planning for lung cancer: how the radiologist can help. Cancer Imaging 2006;6: 116-23 CrossRef Medline

10. Horan G, Roques TW, Curtin J, et al. "Two are better than one": a pilot study of how radiologist and oncologists can collaborate in target volume definition. Cancer Imaging 2006;6:16-19 CrossRef Medline

11. Hollingdale AE, Roques TW, Curtin J, et al. Multidisciplinary collaborative gross tumour volume definition for lung cancer radiotherapy: a prospective study. Cancer Imaging 2011;11:202-08 CrossRef Medline

12. Lee N, Xia P, Fischbein NJ, et al. Intensity-modulated radiation therapy for head-and-neck cancer: the UCSF experience focusing on target volume delineation. Int J Radiat Oncol Biol Phys 2003;57: 49-60 CrossRef Medline

13. O’Neill M, Heron DE, Flickinger JC, et al. Posttreatment quality-oflife assessment in patients with head and neck cancer treated with intensity-modulated radiation therapy. Am J Clin Oncol 2011;34: 478-82 CrossRef Medline

14. Ohri N, Shen X, Dicker AP, et al. Radiotherapy protocol deviations and clinical outcomes: a meta-analysis of cooperative group clinical trials. J Natl Cancer Inst 2013;105:387-93 CrossRef Medline

15. Hartford AC, Galvin JM, Beyer DC, et al American College of Radiology, American Society for Radiation Oncology, Practice guideline for intensity-modulated radiation therapy (IMRT). Am J Clin Oncol 2012;35:612-17 CrossRef Medline

16. Lawrence YR, Whiton MA, Symon Z, et al. Quality assurance peer review chart rounds in 2011: a survey of academic institutions in the United States. Int J Radiat Oncol Biol Phys 2012;84:590-95 CrossRef Medline

17. Grégoire V, Levendag P, Ang KK, et al. CT-based delineation of lymph node levels and related CTVs in the node-negative neck: DAHANCA, EORTC, GORTEC, NCIC, RTOG consensus guidelines. Radiother Oncol 2003;69:227-36 CrossRef Medline

18. Poon I, Fischbein N, Lee N, et al. A population-based atlas and clinical target volume for the head-and-neck lymph nodes. Int J Radiat Oncol Biol Phys 2004;59:1301-11 CrossRef Medline

19. Qazi AA, Pekar V, Kim J, et al. Auto-segmentation of normal and target structures in head and neck CT images: a feature-driven model-based approach. Med Phys 2011;38:6160-70 CrossRef Medline

20. Rosenthal DI, Asper JA, Barker JL Jr, et al. Importance of patient examination to clinical quality assurance in head and neck radiation oncology. Head Neck 2006;28:967-73 CrossRef Medline

21. Ginsberg LE. Reinterpretation of head and neck scans: massive can of worms or call to action? AJNR Am J Neuroradiol 2002;23:1617-18 Medline

22. Zietman A. The future of radiation oncology: the evolution, diversification, and survival of the specialty. Semin Radiat Oncol 2008;18: 207-13 CrossRef Medline

23. Chung NN, Ting LL, Hsu WC, et al. Impact of magnetic resonance imaging versus CT on nasopharyngeal carcinoma: primary tumor target delineation for radiotherapy. Head Neck 2004;26:241-46 CrossRef Medline

24. Wang D, Schultz CJ, Jursinic PA, et al. Initial experience of FDGPET/CT guided IMRT of head-and-neck carcinoma. Int J Radiat Oncol Biol Phys 2006;65:143-51 CrossRef Medline

25. Kovalchuk N, Jalisi S, Subramaniam RM, et al. Deformable registration of preoperative PET/CT with postoperative radiation therapy planning CT in head and neck cancer. Radiographics 2012;32: 1329-41 CrossRef Medline

26. Hwang AB, Bacharach SL, Yom SS, et al. Can positron emission tomography (PET) or PET/computed tomography (CT) acquired in a nontreatment position be accurately registered to a head-andneck radiotherapy planning CT? Int J Radiat Oncol Biol Phys 2009; 73:578-84 CrossRef Medline

27. Sylvester JE, Grimm PD, Eulau SM, et al. Permanent prostate brachytherapy preplanned technique: the modern Seattle method step-by-step and dosimetric outcomes. Brachytherapy 2009;8:197206 CrossRef Medline

28. Barnett GH, Linskey ME, Adler JR, et al; American Association of Neurological Surgeons; Congress of Neurological Surgeons Washington Committee Stereotactic Radiosurgery Task Force. Stereotactic 
radiosurgery: an organized neurosurgery-sanctioned definition. J Neurosurg 2007;106:1-5 CrossRef Medline

29. Erickson B. Image-based brachytherapy: a forum for collaboration between radiation oncologists and diagnostic radiologists. $\mathrm{J} \mathrm{Am}$ Coll Radiol 2005;2:753-58 CrossRef Medline

30. Terezakis SA, Heron DE, Lavigne RF, et al. What the diagnostic radiologist needs to know about radiation oncology. Radiology 2011; 261:30-44 CrossRef Medline

31. Astreinidou E, Bel A, Raaijmakers CP, et al. Adequate margins for random setup uncertainties in head-and-neck IMRT. Int $\mathrm{J}$ Radiat Oncol Biol Phys 2005;61:938-44 CrossRef Medline

32. Siebers JV, Keall PJ, Wu Q, et al. Effect of patient setup errors on simultaneously integrated boost head and neck IMRT treatment plans. Int J Radiat Oncol Biol Phys 2005;63:422-33 CrossRef Medline

33. Hansen EK, Bucci MK, Quivey JM, et al. Repeat CT imaging and replanning during the course of IMRT for head-and-neck cancer. Int J Radiat Oncol Biol Phys 2006;64:355-62 CrossRef Medline

34. Glastonbury CM, Parker EE, Hoang JK. The postradiation neck: evaluating response to treatment and recognizing complications. AJR Am J Roentgenol 2010;195:W164-71 CrossRef Medline

35. Sharma N, Neumann D, Macklis R. The impact of functional imaging on radiation medicine. Radiat Oncol 2008;3:25 CrossRef Medline

36. Newbold K, Partridge M, Cook G, et al. Advanced imaging applied to radiotherapy planning in head and neck cancer: a clinical review. Br J Radiol 2006;79:554-61 CrossRef Medline 